\title{
Seminal volume as an indirect factor for determining success at in vitro fertilization
}

\begin{abstract}
The semen volume is made up by the accessory glands $(90 \%)$ and germinal cells $(10 \%)$ (RB Mayer, 2015). Seminal plasma has enabling factors for sperm microenvironment (Aida Pujol, 2016), allowing among many other processes, sperm maturation (Yu-Wen Kuo, 2016).

Objective: To analyze the correlation of seminal volume parameters with the outcome of fertilized oocytes.

Material and methods: It is a prospective observational study, including 100 couples undergoing in vitro fertilization. The seminal sample was obtained by ejaculation and analyzed with WHO's 2010 criteria (Andrology laboratory procedures manual, 2010). Data encompassed seminal volumes and fertilized oocytes. Statistical analysis was made with Kolmogorov-Smirnov normalization, determining correlation of Pearson and significance of $\mathrm{p} \leq 0.05$
\end{abstract}

Results: The following parameters were registered: Seminal volume: 1.5-7.6 (average 2.7 $\mathrm{ml}$ ), number of fertilized MII oocytes: 1-33 (average 10 oocytes). Pearson correlation of $0.737(p=0.002)$. By dividing groups of fertilized oocytes and seminal volumes by standard Kolmogorov we obtained significance with the number of fertilized eggs with 4.8 and 14 oocytes $(\mathrm{p}=0.001)$, and we also obtained significance with the seminal volume, ranging from 2 to $3.2 \mathrm{ml}(\mathrm{p}=0.003)$

Discussion: Our study shows that it exists a prognostic relationship between the seminal volume and the number of fertilized eggs in an in vitro fertilization. A seminal volume ranging from 2 to $3.2 \mathrm{ml}$ has a better chance to produce adequate fertilization. Volumes lower than this values may reflect inadequate sperm formation and regulation and volumes greater may reflect other inflammatory conditions, such as infection (Jared M Bienek, 2016).

Conclusion: Seminal volume reflects a healthy testicular function, producing spermatozoa capable of achieving good fertilization. Alterations on the seminal volume indicate inadequate conditions for the correct formation of spermatozoa, or increased inflammation molecules that damage the microenvironment.
Volume 6 Issue 3 - 2017

\author{
Hector Salvador Godoy Morales,' Antonio \\ Garcia Luna Vasquez, ${ }^{2}$ Ricardo Mera Mejia, ${ }^{3}$ \\ Stephanie del Rosario Moreno Martinez, ${ }^{2}$ \\ Marcela Josefina Cordova Castillo,' \\ Alejandro Sandoval Garcia Travesi, ${ }^{2}$ Karina \\ Cazarez Perez, ${ }^{2}$ Jose Alfonso Gutierrez \\ Frusch, ${ }^{2}$ Paola Berenice Merchand Alvarez, ${ }^{2}$ \\ Luis Felipe Montano Estrada, ${ }^{2}$ Jose Manuel \\ Lozano Sanchez ${ }^{2}$ \\ 'Director ART Reproducción, Mexico \\ ${ }^{2}$ National Autonomous University of Mexico, Mexico \\ ${ }^{3}$ Biólogo Hospitalángeles Pedregal, Mexico
}

\begin{abstract}
Correspondence: Jose Manuel Lozano Sanchez,ART reproduccion, Camino a Santa Teresa 1055 cp 10700, Te 55434699। I,Email drlozanosachez@gmail.com
\end{abstract}

Received: December 02, 2016 | Published: March 02, 2017
Abbreviations: WHO, World Health Organization; ROS, Source of Reactive Oxygen Species; OS, Oxidative Stress

\section{Introduction}

Infertility incidence in men is difficult to establish accurately. The current evidence suggest that male factor contributes to infertility in $30-50 \%$ of In Vitro Fertilization procedures. ${ }^{1}$ In 1980 , the World Health Organization (WHO) published an international accepted "reference range" to help physicians take appropriate decisions in relation to semen quality. ${ }^{2}$ In the past 30 years this information has been updated constantly, and even though we still haven't got an exact tool to predict efficiently an In Vitro Fertilization prognosis based solely on semen parameters. Besides the genetic information, the contribution that sperm components give to the new embryo and its early development has just begun to be dusted out.

Even though nowadays there are several methods to obtain available sperm to use in an In Vitro Fertilization, most clinics in México and the world rely heavily on ejaculation by masturbation. Previous studies have shown that sperm from the ejaculate may contain higher levels of DNA fragmentation in comparison with sperm obtained directly from the testicle, and even the latter improving outcomes of assisted reproduction. ${ }^{3}$ Seminal plasma can be a source of reactive oxygen species (ROS), which can have a negative impact on the integrity of sperm DNA. ${ }^{4}$ Due to this, it is necessary to know to detail the different components that are part of the ejaculate. The ejaculate is composed of several stages, including the pre-ejaculate phase and the first and second ejaculate fraction. Pre-ejaculate phase contains no sperm, and originates from Cowper and Littré glands, and its objective is to minimize the urethral acidity. The first ejaculate fraction, that consists of $15-45 \%$ of the ejaculate is sperm-rich and contains secretions from the epididymis and prostate. The second ejaculate fraction, consisting of $55-85 \%$ of the entire volume, exhibits a low sperm count and mainly contains seminal gland secretions. ${ }^{4}$ Any problem at any of these stages may affect the environment and the potential fertilization of sperm.

\section{Material and methods}

Our work is a prospective study that encompasses patients from January 2014 to January 2016. We included 100 couples undergoing in vitro fertilization. Seminal samples were obtained by masturbation and analyzed with WHO's 2010 criteria. $^{2}$ They include volumes greater than $1.5 \mathrm{ml}$ with normozoospermia. Samples which contained leucospermia or bacteriospermiawere regarded as infected 
and excluded. We used the semen samples to inseminate MII oocytes by In Vitro Fertilization. The population was statistically normalized with Kolmogorov-Smirnov, and correlate values determined with Pearson, whose significance was set to: $\mathrm{p} \leq 0.05$ The study group was composed by males with body weights between $25-30 \mathrm{~kg}$ (mean $28 \mathrm{~kg}$ ), age 28-38 years (mean 34 years). The study was reviewed by Hospital Angeles Pedregal Ethics committee and all participants accepted and were given written informed consent before their inclusion in the study. This work complies with the Code of Ethics of the World Medical Association (Declaration of Helsinki 1964, revised 2003 and Declaration of Tokyo 1975, revised 2006).

\section{Statistical tests}

We used Spss. v23 for statistical analysis. Kolmogorov-Smirnov was applied for normal distributions, and significance was determined with Pearson correlation $(\mathrm{p} \leq 0.05)$.

\section{Results}

\section{Seminal values baseline parameters}

We obtain 100 semen samples; The values of the seminal parameters showed were $1.5-7.6 \mathrm{ml}(2.7 \mathrm{ml})$ with some significant differences between patients in relation to volume of ejaculation $(1 \mathrm{ml}$ vs $2.7 \mathrm{ml}, \mathrm{p}<0.05)$ and number of fertilized MII ( 1 vs $7, \mathrm{p}<0.01)$. The differences in percentage of volume at ejaculate sample have an important and significant differences between $2 \mathrm{ml}$ volume $(\mathrm{p}=0.002)$ The percentage of oocyte fertilization with sample with significant volume remained unmodified but correlates with the concentration of spermatoids decrease by almost $50 \%(\mathrm{p}=0.0001)$. Nonetheless, the differences between the volumes affect the oocyte fertilization.

\section{Oovocyte fertilization}

The fertilization rate at semen volume under $2.0 \mathrm{ml}$ were decrease and negative correlated with the semen volume sample which the oocyte was use at IVF. It was interesting to observe that the amount of fertilization at MII oocytes depended on the volume in the semen sample.

\section{Clinical associations}

Patients showed a Volume positive correlation with MII (Spearman rho $=0.83, \mathrm{p}<0.001$ ), number of embryos obtained in a cycle (Spearman rho $=0.77, \mathrm{p}=0.007)$ (Table 1) (Figure 1).

Table I Pruebas de normal distribution. Kolmogorov(parametric)

\begin{tabular}{|c|c|c|c|c|c|}
\hline Kolmogorov-Smirnov $^{\mathrm{a}}$ & & & Shapiro-Wilk & & \\
\hline Estadístico & gl & Sig. & Estadístico & gl & Sig. \\
\hline .263 & 4 & . & .902 & 4 & .439 \\
\hline .367 & 4 & . & .729 & 4 & .024 \\
\hline .190 & 6 & $.200 *$ & .919 & 6 & .501 \\
\hline .227 & 4 & . & .944 & 4 & .681 \\
\hline .378 & 3 & . & .766 & 3 & .036 \\
\hline .391 & 4 & . & .700 & 4 & .012 \\
\hline .170 & 5 & $.200^{*}$ & .969 & 5 & .869 \\
\hline .363 & 4 & . & .793 & 4 & .090 \\
\hline .328 & 3 & . & .871 & 3 & .298 \\
\hline .219 & 3 & . & .987 & 3 & .780 \\
\hline .361 & 3 & . & .807 & 3 & .132 \\
\hline .260 & 2 & . & & & \\
\hline .280 & 5 & $.200 *$ & .853 & 5 & .205 \\
\hline .260 & 2 & . & & & \\
\hline .292 & 3 & . & .923 & 3 & .463 \\
\hline
\end{tabular}

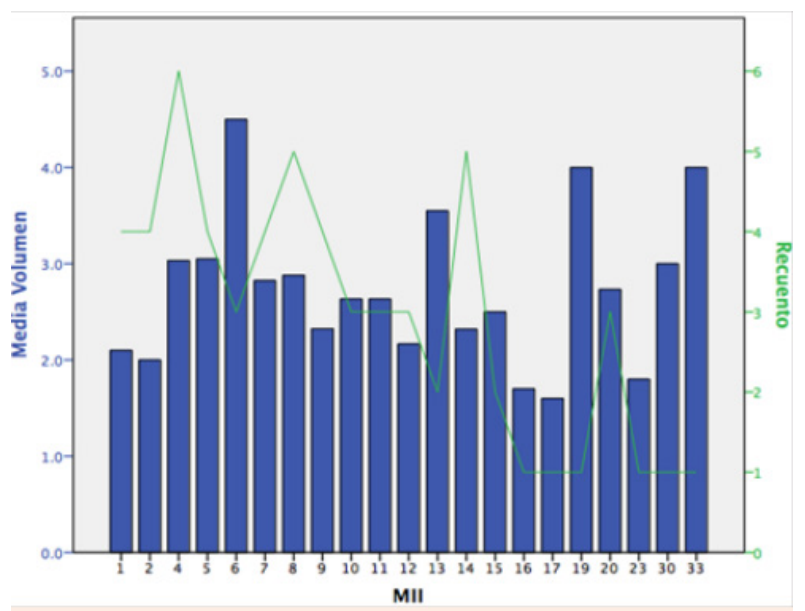

Figure I Patients showed a Volume positive correlation with MII (Spearman rho $=0,83, p<0,00 \mathrm{I})$, number of embryos obtained in a cycle.

\section{Discussion}

There is a positive correlation and statistical significance with the selected sperm sample volume used to inseminate MII eggs and the number of fertilized oocytes by in vitro fertilization technique. A possible explanation for this may be that volumes within this range offer a better microenvironment than larger volumes. Larger volumes are associated with infection and pro-inflammatory factors, and even though we excluded obviously infected semen samples (those reported with leucospermia and bacteriospermia), larger volumes from seminal samples could be associated with increased number of pro-inflammatory substances, as some patients with epididymitis curse with asymptomatic chronic prostatitis.

An in Vitro Fertilization treatment, in order to be successful, requires many variables to be added together and properly. So far, the examination of the male factor and its influence on the results is carried out mainly with seminal morphological parameters. We know that DNA integrity is negatively correlated with infertility and has a direct impact on the quality of the embryo and its development. ${ }^{5}$ 
Damage to DNA is related mainly to oxidative stress (OS). Alterations in the seminal parameters, such as sperm concentration, motility and morphology can be indicative of sperm damage. The link between oxidative stress and bad outcomes in assisted reproduction techniques is based on numerous attacks that occur in cellular metabolism during the process of replication of genetic material and in the regulation of the cell cycle. These sites of regulation, which include G1/S, intra-S and $\mathrm{G} 2 / \mathrm{M}$ are involved in the response to DNA damage. ${ }^{6}$ Zhang et al. ${ }^{7}$ showed that ${ }_{Y} \mathrm{H} 2 \mathrm{AX}$, a marker of DNA damage, appears in H-treated sperm $_{2}$ or $_{2}$ (like OS models) and also embryos fertilized with these sperms have delay for the cleavage, which implies an arrest at the control points of the cell cycle, specifically, G2/M through ATM $\rightarrow$ Chk $1 \rightarrow$ Cdc25B/Cdc25c signaling pathway. ${ }^{7}$

Our results show that the seminal volume of the ejaculate has a statistically significant relationship with adequate fertilization of oocytes in metaphase II. Less than $2 \mathrm{ml}$ volume and a volume greater than $3.2 \mathrm{ml}$ are associated with poor fertilization. We can explain this only from a theoretical point of view, given spermatozoa have no mechanism to repair DNA damage produced by oxidative stress. Sperm use oxidative phosphorylation from mitochondria located in the middle part and Glycolysis in the head and the main part for the movement of flagella and energy production, and at the same time, these processes generate reactive oxygen species (ROS), thus contributing to the formation of oxidative stress and direct injury to sperm's DNA. ${ }^{8}$ There is evidence that increased ROS formation happens in sperm with excess of cytoplasm around the middle part, resulting in dysfunction in sperm's motility and abnormal morphology that impact negatively on the potential of fertilization. ${ }^{9}$ However, the problem is that sperm lacks the appropriate mechanisms to combat the OS and repair the damage caused by it. It requires antioxidants that can prevent, intercept and repair cells from the damage caused by OS. Under normal physiological conditions, the antioxidants help to maintain low levels of oxidative stress in the semen, thus allowing normal processes of cell signaling and functional sperm and preventing cellular damage induced by ROS. ${ }^{10}$

In semen, as well as the intrinsic metabolism of sperm, the main producers of ROS are leukocytes and immature sperm. Leukocytes can produce up to 1,000 times more ROS compared with sperm in normal conditions. ${ }^{11}$ One can find in seminal plasma high concentrations of different enzymatic antioxidants, such as superoxide dismutase, catalase, Glutathione peroxidase, glutathione transferase and ceruloplasmin. ${ }^{12}$ With this evidence, it is clear that proper operation of sperm requires an adequate production of antioxidants in seminal fluid, without which, it could result in damage to the genetic material. However, not all free radicals are aggressive and hostile to sperm cell. There is also conclusive evidence that a proper balance between antioxidants and ROS is necessary. Under normal physiological conditions, adequate levels of ROS are required for sperm maturation, the hyperactivity, and chemotaxis, the union of the pellucid zone, the acrosome reaction and the fusion of the sperm with the egg. ${ }^{10-14}$

Damage to the sperm by OS is not only limited to DNA, multiple studies have demonstrated the association of elevated levels of
ROS with abnormal sperm concentration, motility, morphology and decreased ability of fusion with the oocyte. ${ }^{15}$ Infertile men are more likely to have elevated levels of ROS as a result of its increased production, and not as a decreased concentration of antioxidants when compared against control fertile groups. ${ }^{16}$ Up to $80 \%$ of men with infertility have increased levels of ROS. ${ }^{17}$ However, there are few publications that do not restrict themselves to mentioning the damage directly to the observable sperm in the semen analysis, and focus their efforts to other values such as the characteristics of the seminal plasma and the information that we can provide regardless of the sperm. It is there where this study highlights the importance of the seminal volume of semen samples and its relationship with success in assisted reproduction techniques. Our study returns focus on the importance of seminal volume of samples used for IVF and ICSI obtained through the ejaculate, and reminds us of the importance that all the male genito-urinary system has as a comprehensive and efficient system in order to meet the objective of human reproduction.

\section{Authors' Contributions}

This review demonstrates that seminal volume can be an indirect indicative of the seminal plasma microenvironment. It is clear that there still exists other factors that are not readily studied from semen on an everyday basis, and that there are other tools that clinicians can use as an invaluable aid for the care of patients and helping them achieve their ultimate goal: a healthy baby in home. We still need more studies in order to elucidate the inherent properties of semen and their relationship on the reproductive machinery.

\section{Acknowledgments}

None.

\section{Conflicts of interest}

None.

\section{References}

1. Reed NS, Gomez-Garcia E, Gallardo-Rincon D, et al. Gynecologic Cancer InterGroup (GCIG) consensus review for carcinoid tumors of the ovary. Int J Gynecol Cancer. 2014;24(9 Suppl 3):S35-S41.

2. Behnam K, Kabus D, Behnam M. Primary ovarian undifferentiated non-small cell carcinoma, neuroendocrine type. Gynecol Oncol. 2004;92(1):372-375.

3. Dundr P, Fischerová D, Povýsil C, et al. Primary pure largecell neuroendocrine carcinoma of the ovary. Pathol Res Pract. 2008;204(2):133-137.

4. Yasuda M, Kajiwara H, Osamura YR, et al. Ovarian carcinomas with neuroendocrine differentiation: Review of five cases reffering to immunohistochemical characterisation. J Obstet Gynaecol Res. 2006;32(4):387-395.

5. VerasE,Deavers MT, SilvaEG, etal.Ovariannonsmallcellneuroendocrine carcinoma: a clinicopathologic and immunohistochemical study of 11 cases. Am J Surg Pathol. 2007;31(5):774-782. 